\title{
ARTICLE
}

\section{Cooperative functioning of salicylic acid and phenylalanine ammonia lyase in barley plant challenged with spot blotch and powdery mildew diseases}

\author{
Al-Daoude A.*, Al-Shehadah E., Shoiab A., Jawhar M., Arabi M. I. E. \\ Department of Molecular Biology and Biotechnology, AECS, P.O. Box 6091, Damascus, Syria
}

\begin{abstract}
Salicylic acid (SA) and phenylalanine ammonia-lyase (PAL) have been suggested as important signals during plant resistance towards several fungal pathogens. In this work, to better understand the defense responses initiated by resistant and susceptible barley genotypes challenged with a necrotrophic (Cochliobolus sativus; CS) and a biotrophic (Blumeria graminis; $B g$ ) pathogens, the relative contributions of SA and PAL were investigated at early time points of infection. SA signaling was activated in both genotypes 24 hours post infection (hpi) as compared with the non-inoculated plants. However, with or without pathogen pretreatment, SA significantly increased (P $=0.001$ ) in the resistant genotype that contained three-folds of total SA in comparison with the susceptible one for $B g$. Reverse transcription-polymerase chain reaction (RTPCR) analysis revealed that PAL expression increases in the resistant and susceptible genotypes over the inoculation time points, with the maximum expression observed 48 hpi. PAL expression was paralleled by an increase in SA content in leaves as shown by the test coincidence $\left(F_{3,32}=1.09, P=0.49\right.$ for $C S$ and $F_{3,32}=1.03, P=0.48$ for $\left.B g\right)$. Results showed that the cooperatively function of SA and $P A L$ in barley responses to both $C S$ and $B g$ appeared to be dependent on the plant genotype, and that SA signaling and PAL play a role in barley interactions with these both pathogens. This study might increase our understanding for a deeper molecular research on barley defense responses against pathogens with different lifestyles.

Acta Biol Szeged 63(1):31-36 (2019)
\end{abstract}

\author{
KEY WORDS \\ Hordeum vulgare \\ Blumeria graminis \\ Cochliobolus sativus \\ salicylic acid \\ RT-PCR \\ PAL expression \\ ARTICLE INFORMATION \\ Submitted \\ 17 February 2019 \\ Accepted \\ 16 April 2019. \\ *Corresponding author \\ E-mail: ascientific@aec.org.sy
}

\section{Introduction}

Powdery mildew caused by the obligate biotrophic pathogen, Blumeria graminis f. sp. hordei (Bg), and spot blotch caused by the necrotrophic [Cochliobolus sativus (Cs) Drechs. ex Dastur] are two globally distributed fungal pathogens of barley causing substantial yield losses (Kumar et al. 2002; Rsaliyev et al. 2017). Barley plants respond to both pathogens by activating different mechanisms that are regulated through different plant signaling pathways, including plant hormones such as SA and pathogenesisrelated (PR) proteins (Bindschedler et al. 1998; Jawhar et al. 2017).

Salicylic acid (SA) has been found to regulate the expression of many defense genes (Zwart et al. 2017), which results in altered accumulation of some secondary metabolites. Among them, phenylalanine lyase (PAL) which plays an important role in plant defence and is involved in SA biosynthesis, an essential signal involved in plant systemic resistance (Nugroho et al. 2002; Chaman et al. 2003). Therefore, discovery of SA targets and the understanding of their molecular modes of action in physiological processes could help dissect the SA signaling network, confirming its important role in plant responses to fungal diseases (Vásquez et al. 2015). However, the association between SA and the secondary metabolites synthesized via $P A L$ under fungal pathogen with different lifestyles is unclear. SA effects associated with fungal pathogen infection. However, the role of SA accumulation has not been revealed in full details from the aspect of pathogen lifestyles. Previous works have suggested that plants synthesize SA from phenylalanine (Chen et al. 2009), however, SA could still be produced when this pathway was inhibited. Some bacteria and higher plants are able to synthesize SA using isochorismate synthase (ICS) and pyruvate lyase. In Arabidopsis SA is synthesized from chorismate by means of ICS, and SA made by this pathway is required for local and systemic acquired resistance (Wildermuth et al. 2001).

Reverse transcription-polymerase chain reaction (RT-PCR) is the most reliable technique for measuring the relative expression level of a particular transcript and determining its expression after exposure to a specific 
alteration, such as infection by a fungal pathogen (Bates et al. 2001). In the current work, we studied the defense responses of two barley genotypes Banteng and WI 2291, which are integrated in international breeding programs aimed at developing $C s$ and $B g$ resistant barley genotypes. Banteng was described as a highly resistant to the both pathogens (Arabi and Jawhar 2004, 2012), i.e. exhibited a lower level (compared with WI2291) of Cs and Bg symptom development. We thus hypothesized that SA-triggered defenses could drive contrasted levels of resistance in Banteng and WI2291, inoculated by the same isolate of each pathogen. Thus, the current work aimed at evaluating the changes in SA content and induction of $P A L$ gene expression in two barley cultivars with different levels of resistance towards $C s$ and $B g$ pathogens.

\section{Materials and Methods}

\section{Plant material and experimental design}

The most resistant cv. Banteng and the universal susceptible control cv. WI2291 to all Cs and Bg isolates available for more than 15 years (Arabi and Jawhar 2004, 2012) were used in this study. Seeds of each genotype were planted in plastic boxes $(60 \times 40 \times 8 \mathrm{~cm})$ filled with sterilized peatmoss with three replicates for each pathogen, and each experimental unit consisted of 10 seedlings. They were placed in a growth chamber in a randomized complete block design at temperatures $22^{\circ} \mathrm{C} / 18^{\circ} \mathrm{C}$ (day/night) with 12 -h photoperiod (light intensity was maintained $>200$ $\mathrm{w} / \mathrm{m}^{2}$ ) and $90 \%$ relative humidity.

\section{Infection with Cs}

The most virulent pathotype (Pt4) of C. sativus (Arabi and Jawhar 2004) was used in the experiments. The fungus was incubated in Petri dishes containing potato dextrose agar (PDA, DIFCO, Detroit, MI, USA) for 10 days under $20-21{ }^{\circ} \mathrm{C}$ in the dark. Conidia were collected with sterile distilled water and the suspension was adjusted to $2 \times 10^{4}$ conidia/mL. A surfactant (polyoxyethylene-20-sorbitan monolaurate) was added $(100 \mu \mathrm{L} / \mathrm{L})$ to the conidial suspension to facilitate dispersion of the inoculum over the leaf surfaces. The primary leaves of 12-day-old seedlings were inoculated by uniformly spraying each plant with the conidial suspension using a hand-held spray bottle. C. sativus inoculum preparation, inoculation, post-inoculation and disease records were similar to those described by Fetch and Steffenson (1999) using the percentage of leaf area exhibiting disease symptoms for each genotype were determined using a numerical scale of 1 to 9 with 1 being the most resistant and 9 very susceptible (Fetch and Steffenson 1999). Infection responses 1-3 were deemed resistant, 4-5 moderate, and 6 to 9 deemed susceptible.

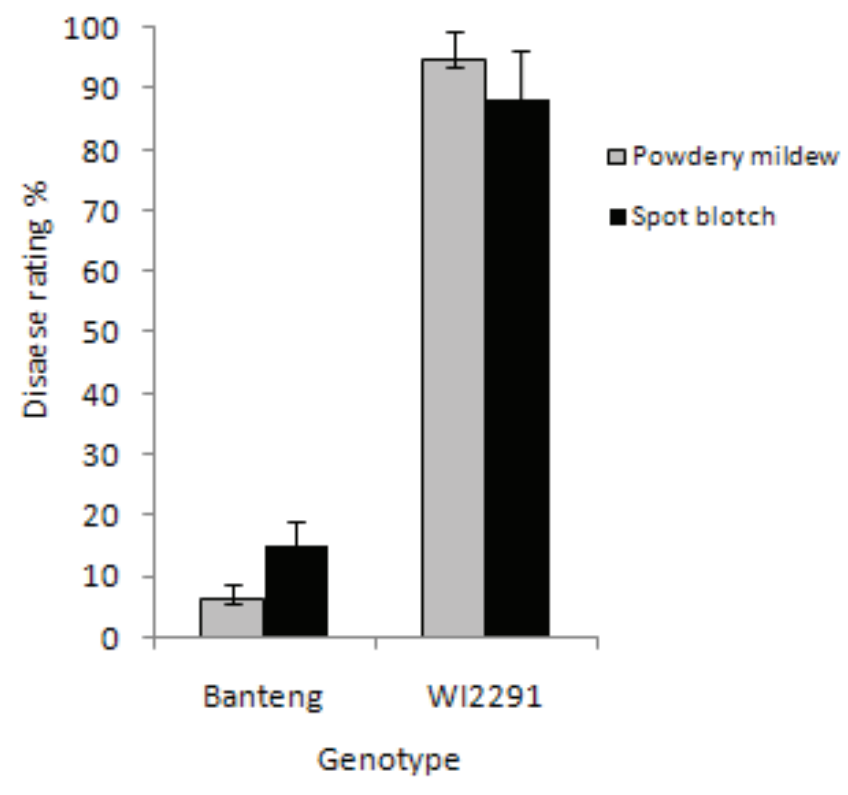

Figure 1. Frequency of disease reactions incited on barley highly resistant genotype Banteng and highly susceptible genotype WI2291 by $C s$ and $B g$. Spot blotch and powdery mildew infections were scored according to Fetch and Steffenson (1999) and Moseman et al. (1981), respectively.

\section{Infection with Bg}

Infection was performed at 12-day-old seedlings following the protocol described by Chaure et al. (2000). Conidiospores of a virulent $\mathrm{Bg}$ isolate were used by employing a soft hair brush to give about 10-20 conidia per one microscope field at $\times 150$ magnification. Inoculated plants were placed under growth chamber conditions, while uninoculated control plants were transferred to a separate "clean" growth chamber and kept under plastic boxes to avoid infection with $B g$. Infections were recorded according to the scale $0-100$ described by Moseman et al. (1981) where: $0=$ no visible symptoms; $100=$ heavy sporulation and all the leaf area covered by a layer of powdery mildew mycelium.

\section{SA quantification}

Pooled samples were prepared from barley leaves taken 24, 48, 72 and 96 hours post inoculation (hpi). For each time point studied, six pooled sample replicates were used for quantification. Free SA was extracted from approximately $200 \mathrm{mg}$ of freshly ground leaves in 1.5 $\mathrm{ml}$ tubes following the method described by Trapp et al. (2014), with minor modifications. Briefly, $100 \mathrm{mg}$ of plant material were dried overnight in a freeze drier at $-42^{\circ} \mathrm{C}$. The extraction was achieved by adding $1.0 \mathrm{~mL}$ of ethyl acetate, dichloromethane, isopropanol, $\mathrm{MeOH}: \mathrm{H}_{2} \mathrm{O}$ into each tube containing dry plant material. Samples were shaken for $30 \mathrm{~min}$ and centrifuged at $16000 \mathrm{~g}$ and 

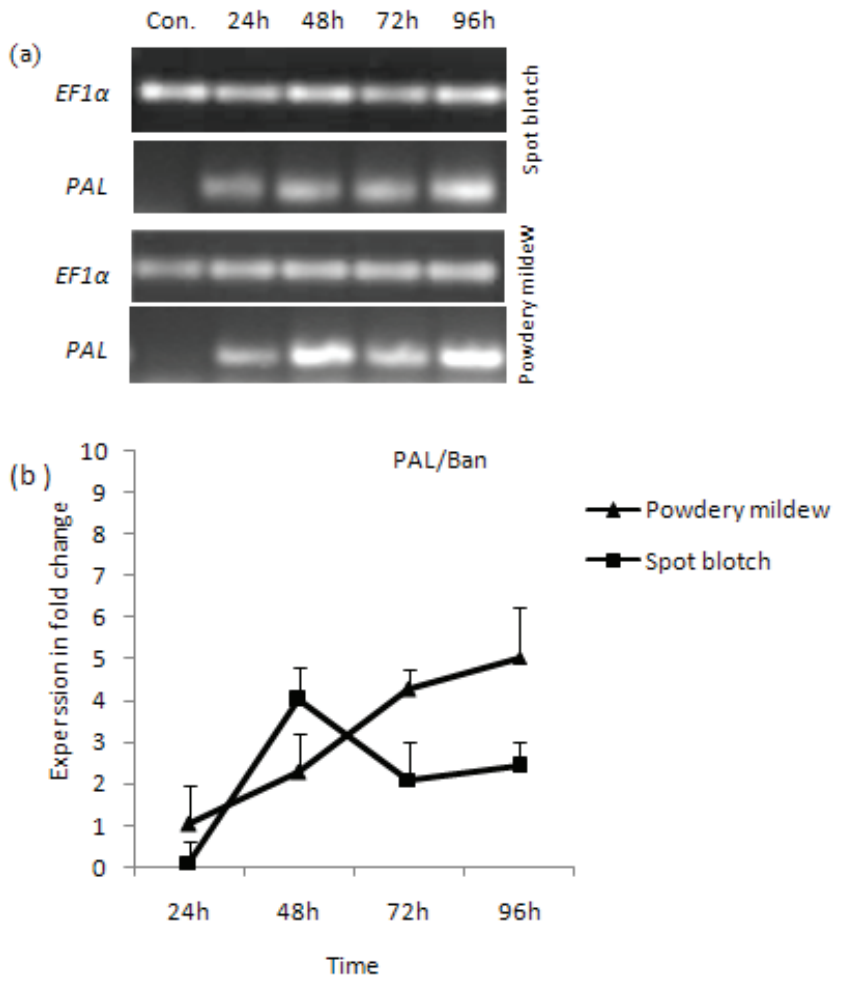

Figure 2. Relative expression profiles of $P A L$ gene in the resistant $\mathrm{cV}$. Banteng, 24, 48, 72 and 96 hours post inoculation with spot blotch and powdery mildew diseases on $1 \%$ agarose gel (a) and its quantification (b).

$4{ }^{\circ} \mathrm{C}$ for $5 \mathrm{~min}$. The supernatant was transferred into a new 1.5 microcentrifuge tube and dried in a speed vac. After drying, $100 \mu \mathrm{L}$ of $\mathrm{MeOH}$ was added to each sample, homogenized under vortex and centrifuged at $16000 \mathrm{~g}$ and $4{ }^{\circ} \mathrm{C}$ for $10 \mathrm{~min}$. SA measurement was performed by a high-performance liquid chromatography (HPLC) system (Agilent Technologies, Germany) connected to a fluorescent detector using an excitation wavelength $\left(\lambda_{\mathrm{EX}}\right)$ of $300 \mathrm{~nm}$ and an emission wavelength $\left(\lambda_{\mathrm{EX}}\right)$ of $410 \mathrm{~nm}$ as described by Verberne et al. (2002). UV detection was performed at $300 \mathrm{~nm}$. Changes in SA content were compared to the control for each time point. Six independent repetitions were performed for each time point. Data were analyzed using the standard deviation and t-test methods.

\section{RNA isolation and CDNA synthesis}

RNA extraction was carried out as described by Cao et al. (1997). Primary leaves of three individual biological replicates collected at 24, 48, 72 and $96 \mathrm{hpi}$ and homogenized with a tube pestle in liquid nitrogen. mRNA was extracted with the Nucleotrap mRNA mini kit (Macherey-Nagel, Germany) following the manufacturer's instructions. RNA was used for cDNA synthesis with the QuantiTect Reverse Transcription Kit (Qiagen) following the manufacturer's instructions. At the same time points, samples from mock inoculated plants were collected as controls. The resulting cDNA products were diluted 20 -fold with autoclaved distilled water, and $2.5 \mu \mathrm{L}$ of the diluted solution was used for quantitative PCR (per). PAL expression was assayed in Step One Plus, 96 well using SYBR Green Master kit (Roche, USA). All cDNA samples, standards and controls (which were tested not to contain genomic DNA) were assayed in triplicate. PCR primers for PAL were designed based on the cDNA sequences of barley available at NCBI (http://www.ncbi.nlm.nih.gov) database (Id: M23548.1) using Primer 3 software. The sequence information for all RT-PCR primers is given in Table 1. The threshold cycle $(\mathrm{Ct})$ value was automatically determined for each reaction by the real time PCR system with default parameters. Seedlings inoculated with distilled water served as a control.

\section{Data analysis}

The fluorescence readings of three replicated samples were averaged, and the blank value (from no-DNA control) was subtracted. Relative expression levels were determined using the average cycle threshold $(\mathrm{Ct})$. Average $\mathrm{Ct}$ values were calculated from the triplicate experiment conducted for each gene, with the $\Delta \mathrm{CT}$ value determined by subtracting the average $\mathrm{Ct}$ value of genes from the $\mathrm{Ct}$ value of the $E F 1 \alpha$ gene. Finally, the equation $2^{-\Delta \Delta \mathrm{CT}}$ was used to estimate relative expression levels (Livak and Schmittgen 2001). Standard deviation was calculated from the replicated experimental data. The statistical analysis was conducted through the Tukey's test at the 0.05 level. The assumption of coincidence was tested using the ANOVA procedure implemented in the software package Statistica 6.1.

Table 1. List of the oligonucleotides used in this study.

\begin{tabular}{lllll}
\hline Gene & Gene description & Accession number & Sequence & Amplified fragment (bp) \\
\hline EF1a & Elongation factor-1 alpha & CV066174 & GGCTGATTGTGCTGTGCTTA (R) & 153 \\
& & & TGGTGGCATCCATCTTGTTA (F) & \\
PAL & Phenylalanine amino lyase & AT2G14610 & CCATTGATGAAGCCAAGCAAG (R) & 123 \\
& & & ATGAGTGGGTTATCGTTGACGG (F) & \\
\hline
\end{tabular}



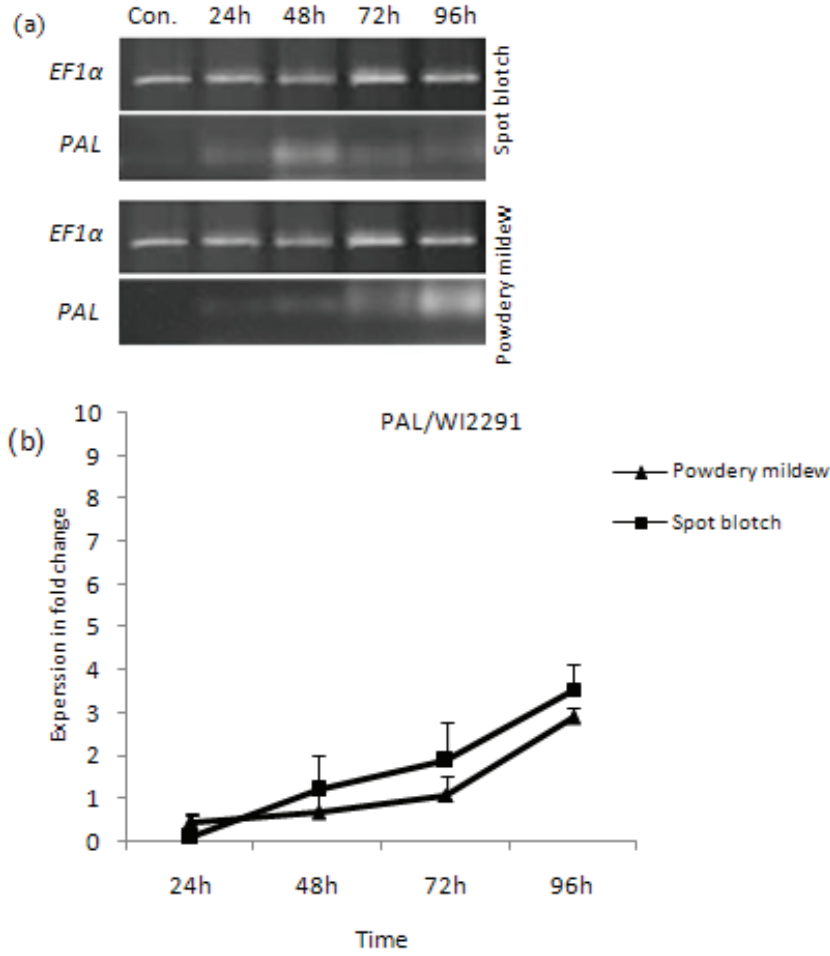

Figure 3. Relative expression of PAL in the susceptible cv. WI2291, 24, 48,72 and 96 hours post infection by spot blotch and powdery mildew diseases on $1 \%$ agarose gel (a) and its quantification (b).

\section{Results and Discussion}

In this study, we used two barley genotypes with different resistant levels to $C s$ and $B g$ infections. As shown in Figure 1, both pathogens caused more severe infection on the susceptible genotype WI2291 as compared with the resistant one Banteng. These results are in agreement with our previous observations under natural field conditions (Arabi and Jawhar 2004, 2012).

Our analysis showed that $P A L$ gene in the resistant barley genotype exhibited a differential expression by $P=0.05$, and were inversely regulated during different time points post inoculation. However at $48 \mathrm{hpi}, P A L$ expression was significantly expressed with 2.4 - and 5.1fold increases, respectively, for barley infected with $B g$ as compared with $C s$. In contrast, $P A L$ was up-regulated 24 hpi and down-regulated $72 \mathrm{hpi}$ in Cs inoculated barley plants as compared to its expression in $B g$ infected plants, suggesting its role at early stages of defense against a biotrophic attack (Fig. 2 and 3). In addition, SA content increased at early time points after pathogen challenge, mainly at 24 hpi in comparison with non-inoculated plants (Fig. 4). It was found that Banteng contained significantly $(P=0.001)$ higher levels of total SA than WI2291 at each time point investigated for both pathogens, which might
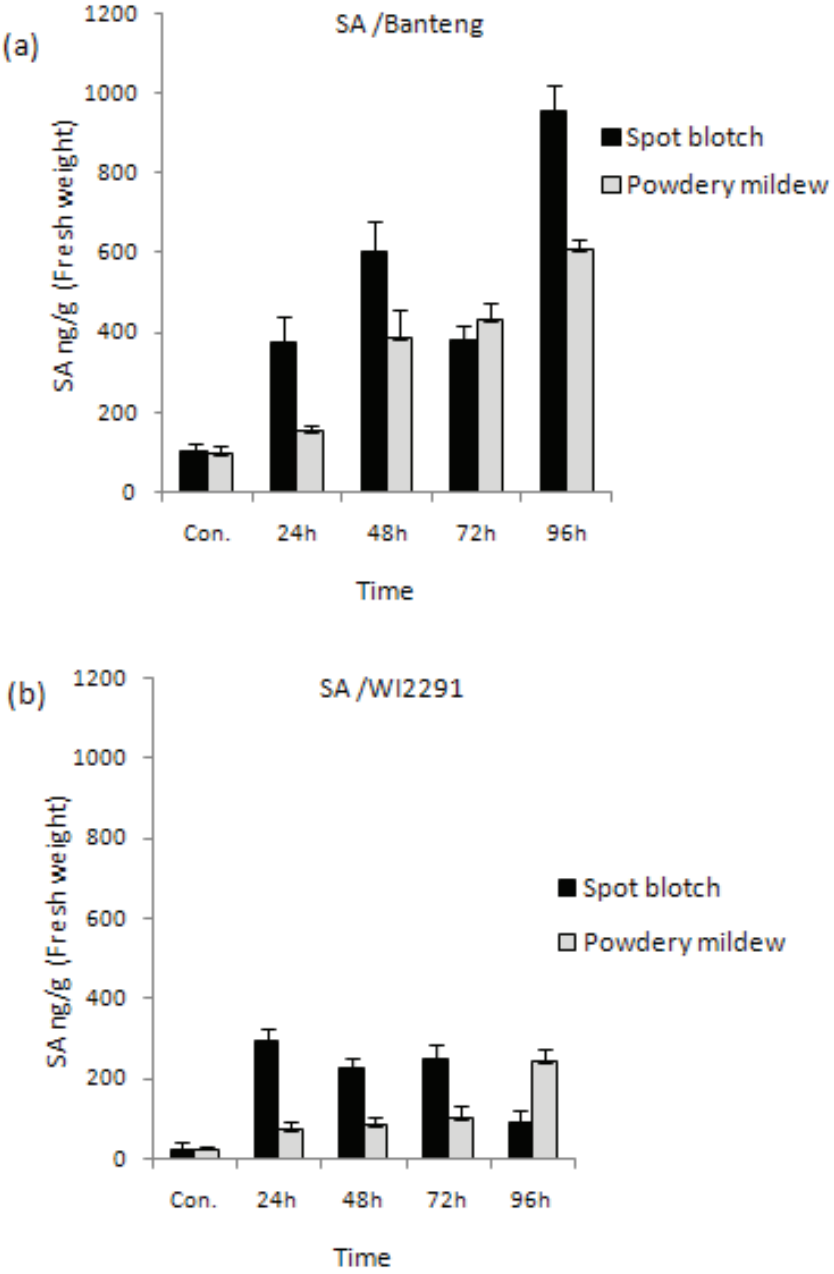

Figure 4. Free salycilic acid quatification in barley leaves post inoculation with spot blotch and powdery mildew in the resistant cv. Banteng (a) and the susceptible cv. WI2291 (b). Error bars represent the stantard error of the means $(n=3)$.

reflect the expected role of SA in signaling events during both $C s$ and $B g$ infection. However, PAL expression was paralleled by an increase in leaf SA content as shown by the coincidence test $\left(\mathrm{F}_{3,32}=1.09, P=0.49\right.$ for $C s$ and $\mathrm{F}_{3,32}=1.03, P=0.48$ for $B g$ ). This is supported by previous works indicating that $P A L$ plays an important role in plant defence; it is involved in the biosynthesis of SA, which is an essential signal involved in plant systemic resistance (Nugroho et al. 2002; Chaman et al. 2003).

RT-PCR expression analysis revealed that the PAL expression increased in the resistant and susceptible genotypes over the inoculation time points for both pathogens, with the highest expression at $96 \mathrm{dpi}$. It has been found that PAL catalyses the non-oxidative deamination of phenylalanine to trans-cinnamate. This is the first step in the phenylpropanoid pathway, and is an important regulation point between primary and sec- 
ondary metabolism (Huang et al. 2010; Vogt 2010). This phenomenon may be the cause of barley cell wall leakage during $C s$ and $B g$ infestations.

The resistant genotype Banteng used for this study was proved to be the most resistant genotype to all isolates of each pathogen available so far (Arabi and Jawhar 2004 2012). The higher activities of $P A L$ and higher level of $\mathrm{SA}$ in infected Banteng leaf tissues compared with the susceptible genotype WI 2291 may explain its high level of resistance. Our finding might support the results of Häffner et al. (2014), who reported that the endogenous SA level in a plant is the main factor of the susceptibility in barley, since pathogen infection may induce plant responses regulated by SA. In addition, SA accumulation was closely associated with redox homeostasis, hypersensitive response, and systemic acquired resistance (Alvarez 2000; Dong 2004).

Moreover, the observed correlation between SA and $P A L$ expression results strengthened when the seedlings were subjected to both $C s$ and $B g$ infections, which is expected given that most secondary metabolites function in improving barley resistance towards both pathogens. One possibility is that SA accumulating in pathogen-infected plants is specifically derived from the PAL isoforms whose expression is induced in the pathogen-infected plants (Chen et al. 2009). However, in contrast to necrotrophs, the biotroph pathogens secrete limited amounts of lytic enzymes, generally lack toxin production and evade detection or suppress immune responses through manipulation of host defenses (Oliver and Ipcho 2004). It has been found that biotrophic Uromyces vignae and the hemibiotrophs Ustilago maydis have suppressed the host defenses during the biotrophic phase (Doehlemann et al. 2008). These findings might support our results when $P A L$ was activated at $24 \mathrm{hpi}$ for $B g$ infection.

This work sheds some light on the relative contributions of SA and PAL during the necrotrophic (Cs) and biotrophic $(B g)$ barley interactions. Results showed that their contribution to the resistance response appears to depend on the plant genotype. It is noteworthy that both SA and PAL have a higher constitutive expression and faster induction in the resistant genotype as compared with the susceptible one. The data demonstrated that the PAL pathway is involved in SA synthesis in barley and more strongly correlated under both pathogens. The obtained results improve our understanding of the manner in which SA regulates secondary metabolites in barley-biotrophic and necrotrophic interactions.

\section{Acknowledgements}

The authors thank the Director General of AECS and the
Head of Molecular Biology and Biotechnology Department for their much appreciated help throughout the period of this research.

\section{References}

Alvarez ME (2000) Salicylic acid in the machinery of hypersensitive cell death and disease resistance. Plant Mol Biol 44:429-442.

Arabi MIE, Jawhar M (2004) Identification of Cochliobolus sativus (spot blotch) isolates expressing differential virulence on barley genotypes in Syria. J Phytopathol 152:461-464.

Arabi MI E, Jawhar M (2012) Expression of resistance to Blumeria graminis in barley genotypes (Hordeum vulgare L.) under field and controlled conditions. J Plant Biol Res 2:107-112.

Bates J, Taylor E, Kenyon D, Thomas J (2001) The application of real-time PCR to the identification, detection and quantification of Pyrenophora species in barley seed. Mol Plant Pathol 2:49-57.

Bindschedler VL, Métraux JP, Schweizer P (1998) Salicylic acid accumulation in barley is pathogen specific but not required for defense-gene activation. Mol Plant-Microbe Inter 11:702-705.

Cao H, Glazebrook J, Clark JD, Volko S, Dong X (1997) The Arabidopsis NPR1 gene that controls systemic acquired resistance encodes a novel protein containing ankyrin repeats. Cell 88:57-64.

Chaman ME, Copaja SV, Argandoña VH (2003) Relationships between salicylic acid content, phenylalanine ammonia-lyase (PAL) activity, and resistance of barley to aphid infestation. J Agric Food Chem 51:2227-2231.

Chaure P, Gurr SJ, Spanu P (2000) Stable transformation of Erysiphe graminis, an obligate biotrophic pathogen of barley. Nat Biotechnol 18:205-207.

Chen Z, Zheng Z, Huang J, Lai Z, Fan B (2009) Biosynthesis of salicylic acid in plants. Plant Sign Behav 4:493-496.

Doehlemann G, Wahl R, Vranes M, De Vries RP, Kämper J, Kahmann R (2008) Establishment of compatibility in the Ustilago maydis/maize pathosystem. J Plant Physiol 165:29-40.

Dong XN (2004) NPR1 all things considered. Curr Opin Plant Biol 7:547-552.

Fetch TG, Steffenson BJ (1999) Rating scales for assessing infection responses of barley infected with Cochliobolus sativus. Plant Dis 83:213-217.

Häffner E, Karlovsky P, Splivallo R, Traczewska A, Diederichsen E (2014) ERECTA, salicylic acid, abscisic acid, and jasmonic acid modulate quantitative disease resistance of Arabidopsis thaliana to Verticillium longisporum. BMC Plant Biol 14:71-85. 
Huang J, Gu M, Lai Z, Fan B, Shi K, Zhou YH, Yu JQ, Chen Z (2010) Functional analysis of the Arabidopsis PAL gene family in plant growth, development, and response to environmental stress. Plant Physiol 153:1526-1538.

Jawhar M, Shoiab A, Arabi MIE, Al-Daoude A (2017) Changes in transcript and protein expression levels in the barley - Cochliobolus sativus interaction. Cereal Res Comm 45:104-113.

Kumar J, Schafer P, Huckelhoven R, Langen G, Baltruschat H, Stein E, Nagarajan S, Kogel HK (2002) Bipolaris sorokiniana, a cereal pathogen of global concern: cytological and molecular approaches towards better control. Mol Plant Pathol 3:185-195.

Livak K J, Schmittgen TD (2001) Analysis of relative gene expression data using real-time quantitative PCR and the 2 (-Delta Delta C (T)) method. Methods 25:402-408.

Moseman JG, Baenziger PS, Kilpatrick RA (1981) Genes conditioning resistance of Hordeum spontaneum to Erysiphe graminins f. sp. hordei. Crop Sci 21(2):229-232.

Nugroho LH, Verberne MC, Verpoorte R (2002) Activities of enzymes involved in the phenylpropanoid pathway in constitutively salicylic acid-producing tobacco plants. Plant Physiol Bioch 40:775-760.

Oliver RP, Ipcho SVSM (2004) Arabidopsis pathology breathes new life into the necrotrophs-vs.-biotrophs classification of fungal pathogens. Mol Plant Pathol
58:347-352.

Rsaliyev A, Pahratdinova Z, Rsaliyev S (2017) Characterizing the pathotype structure of barley powdery mildew and effectiveness of resistance genes to this pathogen in Kazakhstan. BMC Plant Biol 17:178.

Trapp MA, De Souza GD, Filho ER, Boland W, Mithöfer A (2014) Validated method for phytohormone quantification in plants. Front Plant Sci 5:417.

Vásquez AH, Salinas P, Holuigue L (2015) Salicylic acid and reactive oxygen species interplay in the transcriptional control of defense genes expression. Front Plant Sci 6:171.

Verberne MC, Brouwer N, Delbianco F, Linthorst HJM, Bol JF, Verpoorte R (2002) Method for the extraction of the volatile compound salicylic acid from tobacco leaf material. Phytochem Anal 13:45-50.

Vogt T (2010) Phenylpropanoid biosynthesis. Mol Plant 3:2-20.

Wildermuth MC, Dewdney J, Wu G, Ausubel FM (2001) Isochorismate synthase is required to synthesize salicylic acid for plant defence. Nature 29:562-565.

Zwart L, Berger DK, Moleleki LN, van der Merwe NA, Myburg AA, Naidoo S (2017) Evidence for salicylic acid signalling and histological changes in the defence response of Eucalyptus grandis to Chrysoporthe austroafricana. Sci Rep 7:45402. 